\title{
Oral Disease Risk Assessment in Conjunction with Comprehensive Periodontal Care: A Case Report
}

\author{
Hani T Fadel
}

\begin{abstract}
Aim: To illustrate the treatment of a complex periodontal disease patient utilizing oral disease risk assessment.

Background: Periodontal disease is multifactorial in nature and requires addressing multiple factors in order to manage it successfully. Maintenance care is crucial for a favorable outcome, and risk assessment may help formulate the most suitable treatment plan and maintenance program.

Case description: A 37-year-old man presented at the clinic for comprehensive periodontal treatment prior to replacing his missing teeth. He was healthy but smoked 5-10 cigarettes per day. Based on the clinical and radiographic examinations, the patient had generalized advanced periodontitis with multiple caries lesions, defective fillings, and missing teeth. A comprehensive treatment plan was put for the patient and a detailed assessment of his periodontal disease and caries risk was performed. The patient was assigned as being of high risk for periodontal disease progression and of low-medium caries risk. Three months following execution of the proposed nonsurgical and surgical treatment procedures, the patient demonstrated notable improvement compared to the baseline and was put under a strict maintenance program every 3 months.

Conclusion: The presented case illustrates how oral disease risk assessment measures may be incorporated within comprehensive management of a periodontitis patient. Recommending this approach remains a personal preference and is yet to be substantiated by evidence.

Clinical significance: Incorporating risk assessment measures in daily clinical practice may prevent the onset and/or progression of future disease, reducing unnecessary effort and expenses, and should be evaluated by concerned policymakers.

Keywords: Caries risk, Cariogram, Case report, Periodontal risk assessment.

The Journal of Contemporary Dental Practice (2020): 10.5005/jp-journals-10024-2843
\end{abstract}

\section{BACKGROUND}

Optimal periodontal health has major implications on an individual's oral health-related quality of life. ${ }^{1}$ This necessitates that problems affecting the periodontium, i.e., periodontal diseases, should not be left unaddressed.

Traditionally, periodontal treatment focused only on removal of the dental plaque as a sole cause of periodontal disease. Consequently, much attention was given to mechanical and chemical plaque control and removal of (or access to) plaqueretentive areas, and techniques and materials on how to perfect them. This approach proved to be effective on the short-term. ${ }^{2}$ However, several reports pointed to the fact that periodontal treatment without supportive maintenance care is as good as no treatment at all. ${ }^{3}$ A number of complications may arise following periodontal therapy and may jeopardize treatment success. These include caries, root sensitivity, tooth mobility, and dental/ periodontal abscesses. ${ }^{4}$

To add to the complexity, studies have shown that periodontal disease is rather multifactorial in nature, with more than just dental plaque contributing to its occurrence and progression. ${ }^{5}$ It thus requires a much more comprehensive, multifactorial approach for its management than initially proposed. An individual's genetic, systemic, dietary, social, and habitual profiles are only a few patientrelated factors to name on this note. Several local- and site-related factors are also involved as far as pathophysiology is concerned. ${ }^{5}$

Treating the periodontal patient through risk assessment has been proposed in the literature. ${ }^{6} \mathrm{~A}$ few have even advocated the application of risk assessment for other oral diseases such as dental caries as part of the treatment. ${ }^{7}$ It is suggested that this approach would serve as a road map for a more systematic management of
Division of Periodontology, Department of Preventive Dental Sciences, Taibah University Dental College and Hospital (TUDCH), AlMadinah AlMunawwarah, Kingdom of Saudi Arabia

Corresponding Author: Hani T Fadel, Division of Periodontology, Department of Preventive Dental Sciences, Taibah University Dental College and Hospital (TUDCH), AlMadinah AIMunawwarah, Kingdom of Saudi Arabia, Phone: +966 (0) 55440 6095, e-mail: hani.fadel@ yahoo.com

How to cite this article: Fadel HT. Oral Disease Risk Assessment in Conjunction with Comprehensive Periodontal Care: A Case Report. J Contemp Dent Pract 2020;21(5):580-584.

Source of support: Nil

Conflict of interest: None

the different contributory factors, and a guide for maintenance care visits.

In light of the available evidence, the aims of the present report were to illustrate a complex case for a periodontal disease patient who has been treated utilizing oral disease risk assessment methods, and to formulate an acceptable model for managing such cases.

\section{Case Description}

\section{Chief Complaint, Habits, and Health Status}

This case was of a 37-year-old man. He came to the periodontics specialty clinic at the dental school following his general dentist's advice. He wanted to treat his symptomless periodontal condition and then restore his missing back teeth and movable front teeth

(c) The Author(s). 2020 Open Access This article is distributed under the terms of the Creative Commons Attribution 4.0 International License (https://creativecommons. org/licenses/by-nc/4.0/), which permits unrestricted use, distribution, and non-commercial reproduction in any medium, provided you give appropriate credit to the original author(s) and the source, provide a link to the Creative Commons license, and indicate if changes were made. The Creative Commons Public Domain Dedication waiver (http://creativecommons.org/publicdomain/zero/1.0/) applies to the data made available in this article, unless otherwise stated. 
with the least costly fixed treatment option. He noticed occasional bleeding gums when brushing his teeth and he felt tenderness at his cheeks when waking up in the morning.

The patient worked at an insurance company and was of an ectomorphic or slim body build. He ate less than 5 meals/day including snacks, brushed his teeth daily using toothbrush and fluoride toothpaste, and smoked 5-10 cigarettes/day since 15 years. He was systemically healthy, was not on any regular medication, and has not been hospitalized for prolonged periods of time.

\section{Intraoral Clinical Findings}

Examination revealed poor oral hygiene, with generalized calculus deposits and a plaque score of $81 \% .^{8}$ In addition, multiple dental caries lesions, defective restorations, missing teeth $18,26,28,38$, $36,45,47,48$, and overeruption of the upper right second molar, i.e., tooth 17, were observed (Fig. 1A). A soft tissue scar related to the apical area of the upper left lateral incisor, i.e., tooth 22 , was also noted and coincided with an old apical surgery that the patient reported.

Generalized bleeding on probing (BoP $=85 \%)$ and deep periodontal pockets ranging between $6 \mathrm{~mm}$ and $9 \mathrm{~mm}$ were recorded. The attachment loss reached up to $6 \mathrm{~mm}$ in some sites, with grade I-II mobility in upper and lower front teeth. Furcation involvements read classes I and II in upper molar teeth. ${ }^{9}$

\section{Radiographic Picture}

Full-mouth periapical and vertical bite-wing digital images showed generalized horizontal bone loss of 3-7 mm, involving two-third of the root length in some instances (Fig. 2). An apical defect related to tooth 22 was noted. Tooth 14 had apical radiolucency, while tooth 42 had apparent root resorption. Caries, coronal and root fillings, and posts were also observed radiographically.

\section{Diagnosis and Classification}

Based on the case definitions set by 2017 World Workshop on the Classification of Periodontal and Peri-Implant Diseases and Conditions, this case was designated as periodontitis, stage III, grade $\mathrm{C}^{10}$ In addition, the diagnoses of caries at teeth $23 \mathrm{~b}, 24 \mathrm{~b}$, $25 \mathrm{~m}, 37 \mathrm{~b}, 35 \mathrm{~m}, \mathrm{~b}, 34 \mathrm{~b}, 44 \mathrm{~b}, 45 \mathrm{~d}$, apical periodontitis tooth 14, and root resorption tooth 42 were assigned.

\section{Treatment Progress}

Dietary advice as well as recommendation to quit smoking were given. Oral hygiene instructions including the use of a soft toothbrush and interproximal brushes were also stated. Nonsurgical periodontal therapy was performed along with extraction of teeth 14, 32-42, and after reevaluating the case 3 months later (Fig. 1B), resective surgical intervention was carried out at sites with $\geq 6 \mathrm{~mm}$ deep pockets (teeth $24 \mathrm{~m}, 16 \mathrm{~d}$ ). Upon final evaluation of
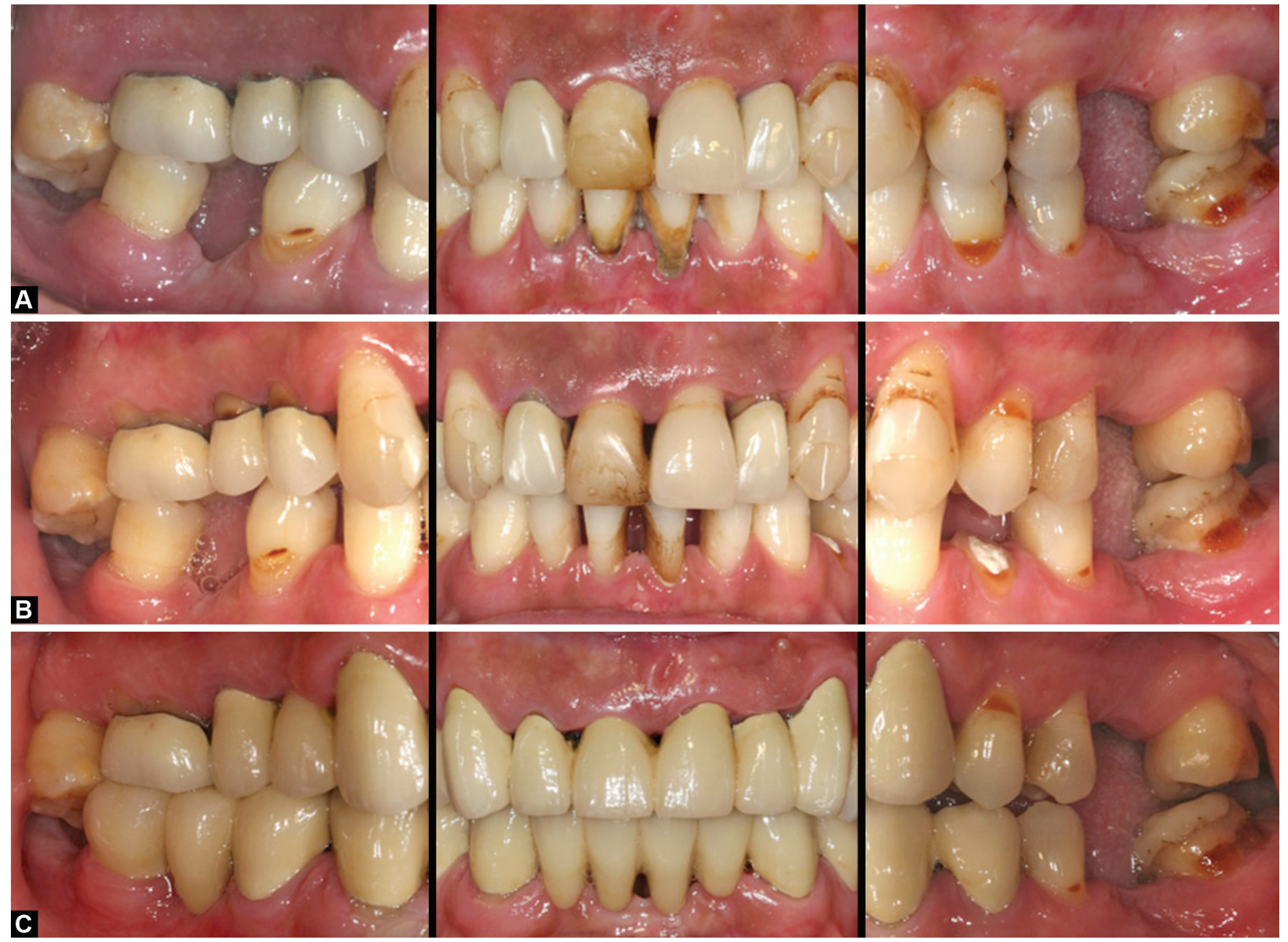

Figs 1 A to C: Right, frontal, and left clinical photographs: (A) Pretreatment; (B) Post-periodontal treatment; (C) Post-prosthodontic treatment 

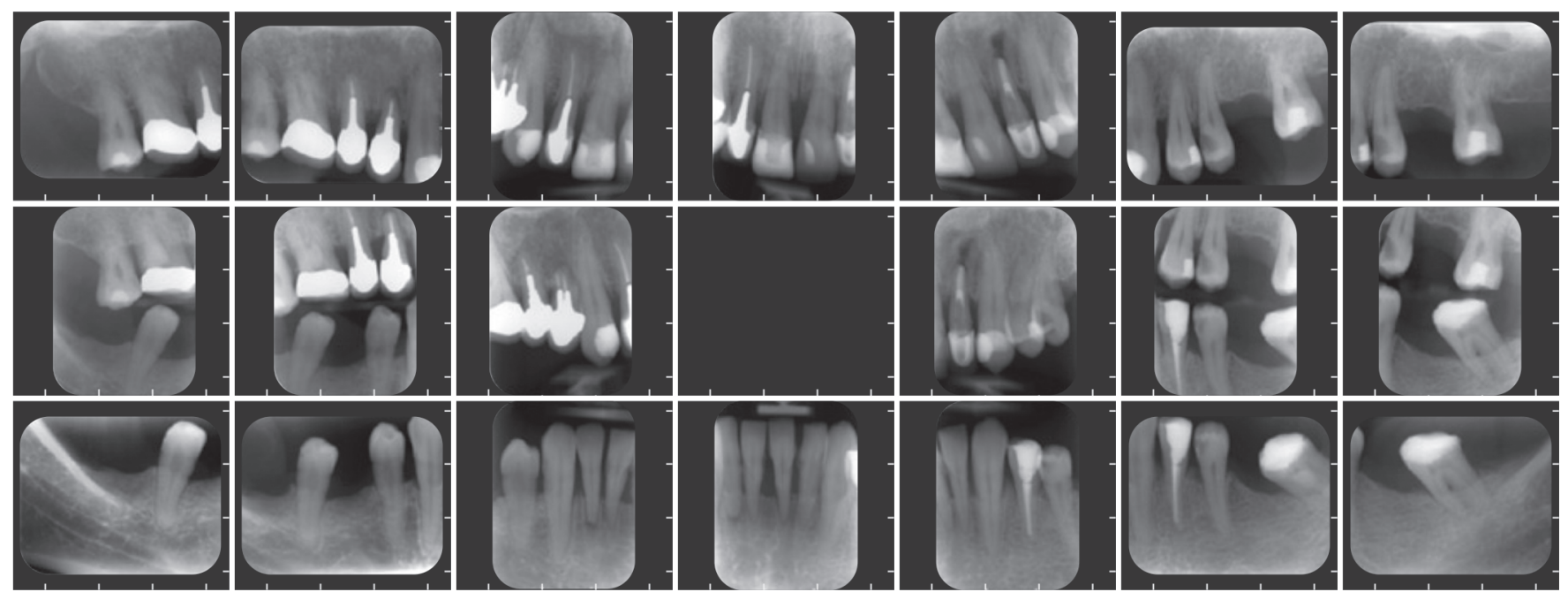

Fig. 2: Pretreatment full-mouth intraoral digital radiographs
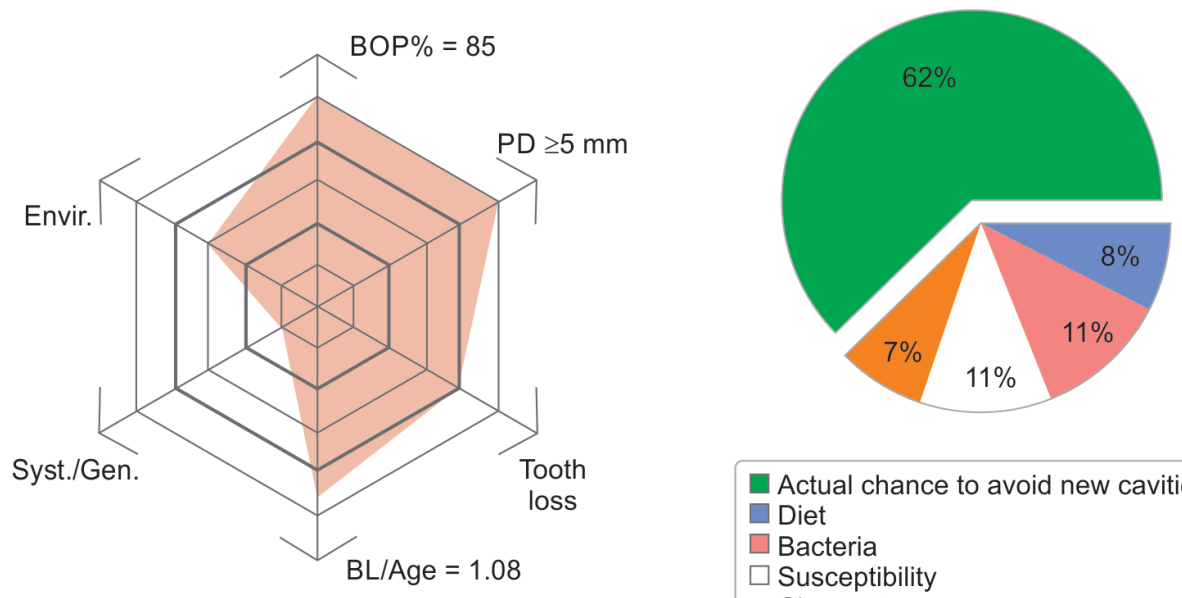

$\square$ Actual chance to avoid new cavities
Diet
$\square$ Bacteria
$\square$ Susceptibility
$\square$ Circumstances

A

B

Figs 3A to B: Risk assessment models: (A) PRA showing high periodontal disease risk; (B) Cariogram showing low-medium caries risk

the case after 3 more months, the periodontal condition improved substantially, with all parameters, i.e., plaque score, bleeding on probing, pocket depth, tooth mobility, decreasing almost threefold. Cross-arch, fixed dental prostheses were placed in the upper and lower arches to replace missing teeth 14, 32-42, 45, and defective restorative work at teeth $15,13,12,22,23,35$, and 44 (Fig. 1C). Other prosthodontic options were discussed such as dental implant therapy. However, the finally selected option was reached based on the patient wishes, economic status, and prosthodontist's advice.

In total, the treatment lasted for 8 months. Following the initial visit, nonsurgical periodontal treatment and caries control were carried out (1 month), followed by the first reevaluation and surgical periodontal treatment (3 months). The second reevaluation was then done (3 months), followed by the final prosthetic work (1 month).

\section{Periodontal and Caries Risk Assessment}

The periodontal risk assessment (PRA) polygon was used when formulating the treatment plan. ${ }^{11}$ A number of local and patientrelated factors were used to determine the patient's risk for periodontal disease progression. These included the individual's age, general health status, smoking habits, number of lost teeth, sites with pocket depth $\geq 5 \mathrm{~mm}$, bleeding on probing, and radiographic alveolar bone loss. Based on the PRA, the patient was considered of high risk for progression, indicated by the polygon surface area and the number of factors reaching the higher limits (Fig. 3A). Similarly, the cariogram was used to assess the patient's caries risk. ${ }^{12}$ Nine caries-related factors were assessed, including the caries experience, current medication, eating habits, fluoride practices, dental plaque accumulation, and saliva samples to assess the salivary flow rate, buffer capacity, and cariogenic bacterial counts. The stimulated salivary secretion rate was determined after having the patient chew on a piece of paraffin wax for 1 minute till it softened then spit in a measurement tube while chewing for 5 minutes. ${ }^{13}$ The salivary buffering capacity was determined by analyzing the saliva sample according to the method described by Ericsson. ${ }^{14}$ Counts of cariogenic bacteria, that is, mutans streptococci and lactobacilli, on the other hand, were determined using basic agar plates. ${ }^{15,16}$ Accordingly, the patient was considered of low to medium caries risk, indicated by the large green sector of the resulting pie chart named "Actual Chance to Avoid New Cavities" (Fig. 3B). 


\section{Outcome and Maintenance Care}

Based on the assessments by the PRA and the cariogram, the patient was put on a maintenance program every 3 months for a year and onward. During maintenance visits, medical history, dietary and daily habits, and personal oral hygiene practices were regularly updated. The patient was also asked about any changes in his oral health status or newly risen complaints related to his teeth and gums. Clinical examinations were routinely performed, while radiographs were taken only when indicated. Oral hygiene instructions were reinforced and oral prophylaxis and fluoride application were done at every recall. Any newly formed calculus was selectively removed.

The patient was satisfied with the final outcome of the treatment and complied with the proposed recall program, as he understood the importance of the follow-up visits for the sustainability of his oral condition.

\section{Discussion}

This report presents a case of a patient requiring comprehensive periodontal and prosthodontic care. It is well established that supportive care is the key for successful periodontal therapy. ${ }^{17}$ Classical studies have demonstrated the benefit of long-term maintenance on periodontal treatment outcome. ${ }^{18}$ Zafiropoulos et al. showed how a case was deteriorating periodontally and around implants due to lack of regular maintenance. ${ }^{19}$ Moreover, tooth loss has been clearly linked to the frequency of maintenance care. ${ }^{20}$

The latest classification of periodontal and peri-implant diseases was used for the current case. ${ }^{10}$ This helped for a more treatment-oriented approach when assigning the diagnosis as originally aimed for by the classification taskforce. Walter and coworkers have also adopted the latest classification in their report of an advanced periodontitis case and mentioned the benefits pertaining to the proposed treatment strategy and maintenance care compared to previous classification systems. ${ }^{21}$

Periodontal and caries risk assessment was used as part of the current case management. Assessment of periodontal disease risk may be of great value in treated populations. ${ }^{6}$ Several studies utilizing risk assessment have significantly predicted treatment outcome in terms of tooth loss ${ }^{22}$ and periodontitis progression. ${ }^{23}$ However, this necessitates the continuous performance of the risk assessment procedure, as the patient status changes over the course of treatment. ${ }^{24}$ Nevertheless, the benefits of following a systematic approach and accounting for individual risk factors have been illustrated in a number of reports. ${ }^{25,26}$

The assessment of an individual's caries risk during periodontal therapy has been proposed by a number of researchers. ${ }^{7,27} \mathrm{~A}$ plausible explanation could be the resulting gingival recession following periodontal treatment and the consequent root surface exposure. ${ }^{28}$ As it is well known that root surfaces are more prone to caries than the tooth crown, more necessary care in case of a high caries-risk individual is recommended. ${ }^{29}$ In addition, the microbial shifting from a periodontopathic to a more cariogenic environment following periodontal therapy also points toward the need for detecting those individuals who exhibit high caries risk. ${ }^{30,31}$

\section{Conclusion}

To conclude, the presented case illustrates how oral disease risk assessment measures may be incorporated within comprehensive management of a periodontitis patient. Despite the potential benefits, practitioners may neglect routine use of risk assessment in daily practice due to the extra time and effort needed. Therefore, such a recommendation remains as a personal preference and is yet to be substantiated.

\section{Clinical Relevance}

The incorporation of risk assessment measures within daily clinical practice may aid in preventing the onset and/or progression of future disease. This could help reduce unnecessary effort and expenses both on the dentist and the patient, and could be an approach worth evaluating by officials and concerned policymakers.

\section{References}

1. Ferreira MC, Dias-Pereira AC, Branco-de-Almeida LS, et al. Impact of periodontal disease on quality of life: a systematic review. J Periodontal Res 2017;52(4):651-665. DOI: 10.1111/jre.12436.

2. Fardal O, Linden GJ. Re-treatment profiles during long-term maintenance therapy in a periodontal practice in Norway. J Clin Periodontol 2005;32(7):744-749. DOI: 10.1111/j.1600051X.2005.00778.x.

3. Becker W, Becker BE, Berg LE. Periodontal treatment without maintenance. A retrospective study in 44 patients. J Periodontol 1984;55(9):505-509. DOI: 10.1902/jop.1984.55.9.505.

4. Renvert S, Persson GR. Supportive periodontal therapy. Periodontol 2000 2004;36:179-195. DOI: 10.1111/j.1600-0757.2004.03680.x.

5. Pihlstrom BL, Michalowicz BS, Johnson NW. Periodontal diseases. Lancet 2005;366(9499):1809-1820. DOI: 10.1016/S01406736(05)67728-8.

6. Lang NP, Suvan JE, Tonetti MS. Risk factor assessment tools for the prevention of periodontitis progression a systematic review. J Clin Periodontol 2015;42(Suppl 16):S59-S70. DOI: 10.1111/jcpe.12350.

7. Ravald N, Birkhed D, Hamp SE. Root caries susceptibility in periodontally treated patients. Results after 12 years. J Clin Periodontol 1993;20(2):124-129. DOI: 10.1111/j.1600-051X.1993. tb00326.x.

8. O'Leary TJ, Drake RB, Naylor JE. The plaque control record. J Periodontol 1972;43(1):38. DOI: 10.1902/jop.1972.43.1.38.

9. Hamp SE, Nyman S, Lindhe J. Periodontal treatment of multirooted teeth. Results after 5 years. J Clin Periodontol 1975;2(3):126-135. DOI: 10.1111/j.1600-051X.1975.tb01734.x.

10. Tonetti MS, Greenwell H, Kornman KS. Staging and grading of periodontitis: framework and proposal of a new classification and case definition. J Clin Periodontol 2018;45(Suppl 20):S149-S161. DOI: 10.1111/jcpe.12945.

11. Lang NP, Tonetti MS. Periodontal risk assessment (PRA) for patients in supportive periodontal therapy (SPT). Oral Health Prev Dent 2003;1(1):7-16.

12. Bratthall D, Hänsel Petersson G. Cariogram--a multifactorial risk assessment model for a multifactorial disease. Community Dent Oral Epidemiol 2005;33(4):256-264. DOI: 10.1111/j.16000528.2005.00233.x.

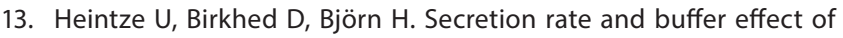
resting and stimulated whole saliva as a function of age and sex. Swed Dent J 1983;7(6):227-238.

14. Ericsson Y. Clinical investigations of the salivary buffering action. Acta Odontol Scand 1959;17(2):131-165. DOI: 10.3109/00016355908993928.

15. Gold OG, Jordan HV, Van Houte J. A selective medium for streptococcus mutans. Arch Oral Biol 1973;18(11):1357-1364. DOI: 10.1016/0003-9969(73)90109-X.

16. Rogosa M, Mitchell JA, Wiseman RF. A selective medium for the isolation and enumeration of oral and fecal lactobacilli. J Bacteriol 1951;62(1):132-133. DOI: 10.1128/JB.62.1.132-133.1951.

17. McClain PK. Maintenance: the key to successful periodontal and implant therapy. Compend Contin Educ Dent 2014;35(3 Suppl):4-10. quiz $1,7$. 
18. Axelsson $P$, Nystrom B, Lindhe J. The long-term effect of a plaque control program on tooth mortality, caries and periodontal disease in adults. Results after 30 years of maintenance. J Clin Periodontol 2004;31(9):749-757. DOI: 10.1111/j.1600-051X.2004. 00563.x.

19. Zafiropoulos GG, Parashis A, Abdullah T, et al. Comprehensive treatment of severe periodontal and periimplant bone destruction caused by iatrogenic factors. Case Rep Dent 2018;2018:7174608. DOI: 10.1155/2018/7174608.

20. Farooqi OA, Wehler CJ, Gibson G, et al. Appropriate recall interval for periodontal maintenance: a systematic review. J Evid Based Dent Pract 2015;15(4):171-181. DOI: 10.1016/j.jebdp.2015.10.001.

21. Walter C, Chapple ILC, Ower P, et al. Periodontal diagnosis in the context of the BSP implementation plan for the 2017 classification system of periodontal diseases and conditions: presentation of a patient with severe periodontitis following successful periodontal therapy and supportive periodontal treatment. Br Dent J 2019;226(6):411-413. DOI: 10.1038/s41415-019-0143-8.

22. Eickholz P, Kaltschmitt J, Berbig J, et al. Tooth loss after active periodontal therapy. 1: patient-related factors for risk, prognosis, and quality of outcome. J Clin Periodontol 2008;35(2):165-174. DOI: 10.1111/j.1600-051X.2007.01184.x.

23. Matuliene G, Studer R, Lang NP, et al. Significance of periodontal risk assessment in the recurrence of periodontitis and tooth loss. J Clin Periodontol 2010;37(2):191-199. DOI: 10.1111/j.1600051X.2009.01508.x.
24. Tan AE. Periodontal maintenance. Aust Dent J 2009;54(Suppl 1): S110-S117. DOI: 10.1111/j.1834-7819.2009.01149.x.

25. Afshar A. Systematic risk management: providing a patient the smile she long desired. Compend Contin Educ Dent 2015;36(8):582-587.

26. Kois DE, Kois JC. Comprehensive risk-based diagnostically driven treatment planning: developing sequentially generated treatment. Dent Clin North Am 2015;59(3):593-608. DOI: 10.1016/ j.cden.2015.03.001.

27. Reiker J, van der Velden U, Barendregt DS, et al. A cross-sectional study into the prevalence of root caries in periodontal maintenance patients. J Clin Periodontol 1999;26(1):26-32. DOI: 10.1034/j.1600051X.1999.260105.x.

28. Tugnait A, Clerehugh V. Gingival recession-its significance and management. J Dent 2001;29(6):381-394. DOI: 10.1016/S03005712(01)00035-5.

29. Rodrigues JA, Lussi A, Seemann R, et al. Prevention of crown and root caries in adults. Periodontol 2000 2011;55(1):231-249. DOI: 10.1111/j.1600-0757.2010.00381.x.

30. Quirynen M, Gizani S, Mongardini C, et al. The effect of periodontal therapy on the number of cariogenic bacteria in different intra-oral niches. J Clin Periodontol 1999;26(5):322-327. DOI: 10.1034/j.1600051X.1999.260511.x.

31. Quirynen M, Listgarten MA. Distribution of bacterial morphotypes around natural teeth and titanium implants ad modum Branemark. Clin Oral Implants Res 1990;1(1):8-12. DOI: 10.1034/j.16000501.1990.010102.x. 